\title{
SIMULATIONS OF RACETRACK MICROTRON FOR ACCELERATION OF PICOSECOND ELECTRON PULSE ${ }^{*}$
}

\author{
M. Washio ${ }^{\#}$, R. Kuroda, Waseda University, 3-4-1 Okubo, Shinjuku, Tokyo \\ J. Yang. T. Hori , F. Sakai, Sumitomo Heavy Industries Ltd.,1-1-2 Yato-cho, Tanashi, Tokyo
}

\section{Abstract}

Low emittance sub-picosecond electron pulses are expected to be used in a wide field, such as free electron laser, laser acceleration, femtosecond X-ray generation by inverse Compton scattering, and pulse radiolysis, etc. In order to produce the low emittance sub-picosecond electron pulses, we are developing a compact racetrack microtron (RTM) with a new $5 \mathrm{MeV}$ injection system adopting an laser photo cathode RF gun [1]. The operation of RTM is kept under the steady state of beam loading for long pulse mode so far[2]. We have investigated for the first time by numerical simulation in the case of short- and single-pulse acceleration. As the results, RTM is also useful to accelerate a picosecond electron pulse under a transient state of beam loading. In the simulation, a picosecond electron pulse is accelerated to $139 \mathrm{MeV}$ in RTM for the injection of about $5 \mathrm{MeV}$ pulse with pulse length of 3 picoseconds, charge of $1 \mathrm{nC}$ per pulse, and emittance of $1.8 \pi \mathrm{mm} \mathrm{mrad}$, which corresponds of output of the RF gun.

\section{INTRODUCTION}

Ultra-short and low emittance electron beams are indispensable tool for the physical chemistry investigation in ionisation and excitation processes of various kind of materials. Further, high quality X-ray beam with the pulse length of the pico- to femtosecond time region can be generated by the Inverse Compton Scattering process between high-brightness and short pulse laser light and the high quality electron beam [1-3]. One of the most powerful methods to generate the high quality electron beam is considered to apply a photo cathode RF gun system in the combination with racetrack Microtron (RTM). Recent rapid progress of the photo cathode RF gun system conducted by the collaboration among the BNL, KEK and SHI[4] promises us to generate suitable electron beams to inject into the RTM with desired beam parameters. In such a way, we can generate short pulse and very low emittance electron beam with the energy up to $150 \mathrm{MeV}$ [5].

The design of RTM has been established and been demonstrated as the injectors of compact SR rings, AURORA-1 and-2[6]. The already-existing design is, however, optimized for the output beam having

"Work supported in part by the Grant for Special Project of Waseda University, No. 98A-901

"Email: washiom@mn.waseda.ac.jp somewhat long electron pulse length at around a few microseconds and relatively low peak current, $10 \mathrm{~mA}$ at the maximum. When we apply the combination of RTM with photo cathode RF gun as the injection system, we have to investigate the behaviours of electron beams on the condition of transient beam loading and effect of chicane magnets for the $5 \mathrm{MeV}$ electron injection. The system configuration is shown in Fig. 1. The effects of space charge and synchrotron radiation while acceleration would next be taken into account.

In the first step of the simulation, we have calculated the output beam characteristics obtained from photo cathode RF gun using MAGIC code. In the second step, we have calculated the final beam characteristics as the output from RTM using a modified SUPERFISH code to treat the time dependent acceleration field.

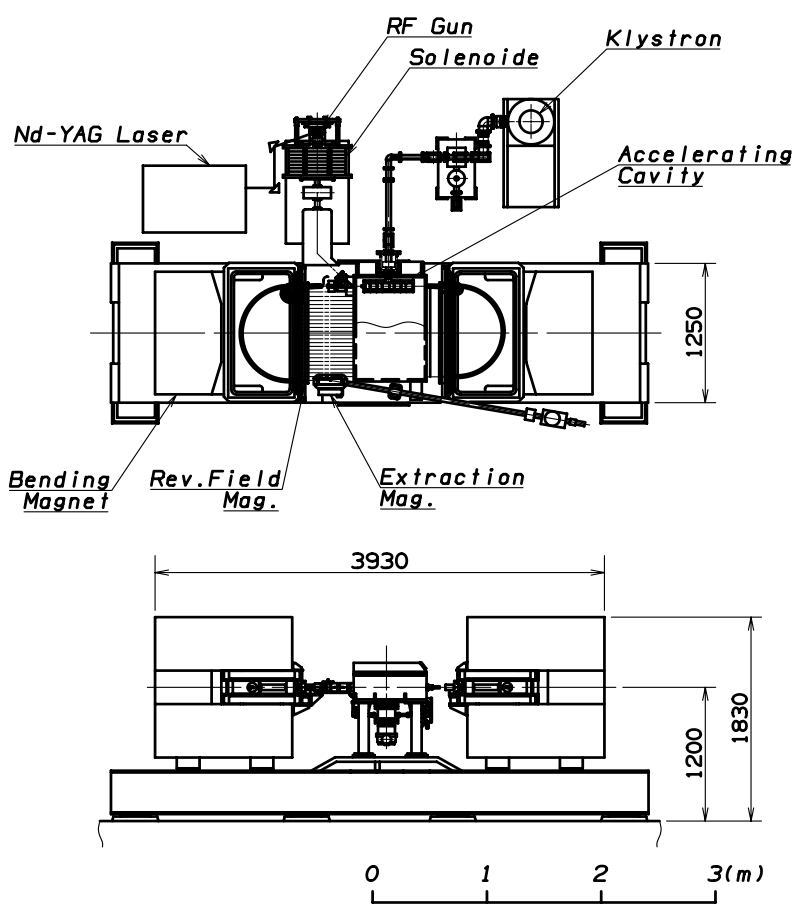

Figure 1 : Top view of 150-MeV RTM with RF gun injection system

\section{RF GUN}

$\mathrm{RF}$ gun using for our simulation is based on so-called BNL type, 1.6 cell s-band cavity structure. Fig 2 shows the typical emittance result obtained for the gun using the following parameters. 
- Input laser pulse length : 10ps

- Laser beam spot size in diameter : $2.4 \mathrm{~mm}$

- Acceleration Field Strength : 100MV/m

- Resonance Frequency : $2854.62 \mathrm{MHz}$ as a $\pi$ mode

- Energy Gain : $4.9375 \pm 0.0225 \mathrm{MeV}$

- Electron Charge : $100 \mathrm{pC}$

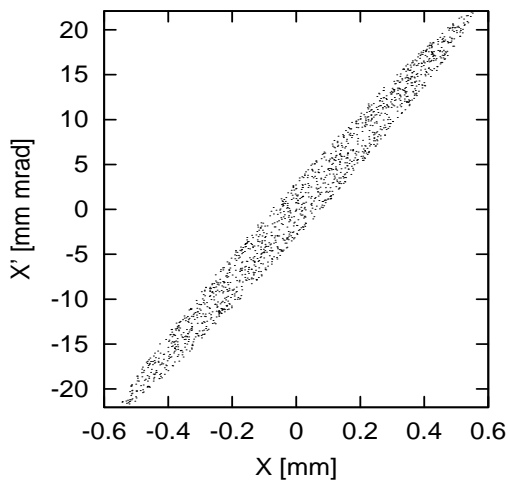

Figure 2 : Normalized emittance of output beam from RF gun

The obtained normalized rms emittance is about $1.8 \mathrm{~mm}$ mrad. with the pulse length of $3 \mathrm{ps}$. This emittance value is not so small in the view point of RF gun characteristics. Better emittance can be obtained using other parameters by selecting the injection RF phase and laser light diameter, in the electron charge up to several hundreds of $\mathrm{pC}$, however, acceptance of RTM is much limited and we selected the above parameters for the complete acceptance to RTM

\section{MICROTRON}

The major components of RTM are two 180 degree bending magnets placed on both end-sides with reverse field one in front of each, one s-band accelerating structure of $0.5 \mathrm{~m}$ long placed on the mid-portion of the first orbit near the injection point, and the RF gun as the injector. Here, except this new injection scheme, other sub-systems are precisely the same as the normal parameters of $150 \mathrm{MeV}$ RTM. This means that the optimisation of parameters for high energy and low emittance electron injection does not carried out in this study. The principal parameters of RTM for the numerical calculation are shown in Table 1.

\begin{tabular}{|l|l|}
\hline Circulating No. & $23 \quad$ laps \\
Energy Gain & $6 \quad$ MeV/lap \\
Bending Field & 1.23 and 1.228 Tesla \\
Field Gradient & $0.14 \quad$ Tesla/m \\
Reverse Field & 0.2919 and 0.2777 Tesla \\
Chicane Field & $0.33 \quad$ Tesla \\
RF Frequency & $2856 \mathrm{MHz}$ \\
Accelerating Gradient & $15 \quad \mathrm{MV} / \mathrm{m}$ \\
No. of Accelerating Cell & 7 full +2 half cells \\
\hline
\end{tabular}

Table 1: Parameters of RTM

\section{SIMULATION RESULTS}

For the simulation of single-bunch of $1 \mathrm{nC}$, we have roughly estimated the stored energy in the acceleration cavity before and after the single-bunch acceleration. Originally, there is about 2 joules of stored energy in the cavity which consists of 7-full and 2-half cells of side coupled type. During the acceleration of $1 \mathrm{nC}$ electron bunch, it needs about 0.15 joule when accelerated to 150 $\mathrm{MeV}$. Thus, about $7.5 \%$ of stored energy is taken away by the beam which enforces about $3.8 \%$ decline of electric field upon the cavity when no refill of RF power is assumed.

We have calculated the beam transmittance while circulating in RTM and the emittance after the acceleration are shown in Figs. 3 and 4 using the input parameters of Fig.2. Beam transmittance data shows that about $82 \%$ of electrons are obtained at the acceleration energy to $139.55 \mathrm{MeV}$. The emittance data after the acceleration, we have obtained rms- $\varepsilon x,-\varepsilon y$ are 0.12 and $0.037 \pi \mathrm{mm}$ mrad., respectively. These are the smallest values which we have obtained by experiments and calculations[5], however, these are still larger than expected values.

The distribution of $(\mathrm{E}, \phi)$ phase space of accelerated electrons within a bunch are shown in Fig. 5 (right) together with the histogram of energy distribution (left). From the figure, the beam energy after 23 laps of acceleration is read as $139.55 \mathrm{MeV}$ and energy spread $\Delta \mathrm{E} / \mathrm{E}$ as $\pm 0.07 \%$. The phase distribution is more clearly shown in Fig.6, where the final distribution are demonstrated. The phase spread is compressed to $1.5^{\circ}$ after the acceleration, about a half of the initial spread. It is equivalent to $1.5 \mathrm{psec}$ pulse length. When acceleration is performed up to $151 \mathrm{MeV}$, we can get smaller energy spread down to $\pm 0.02 \%$ of $\Delta \mathrm{E} / \mathrm{E}$.

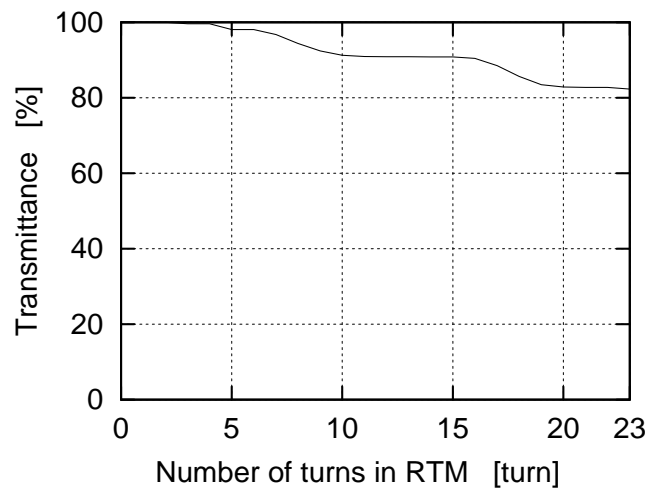

Figure 3 : Beam transmittance in RTM 

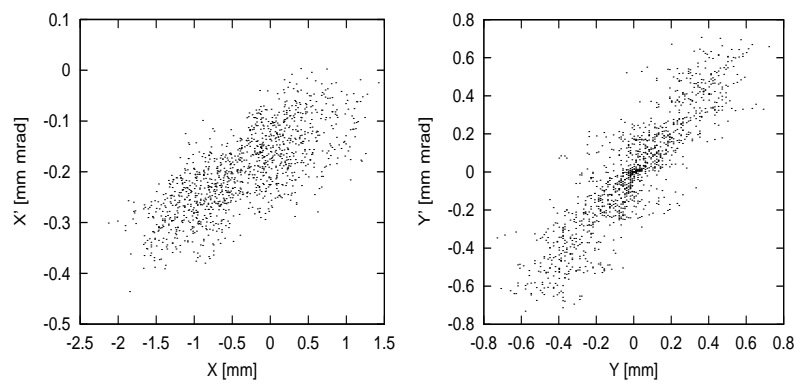

Figure 4 : Final emittance of output beam from RTM
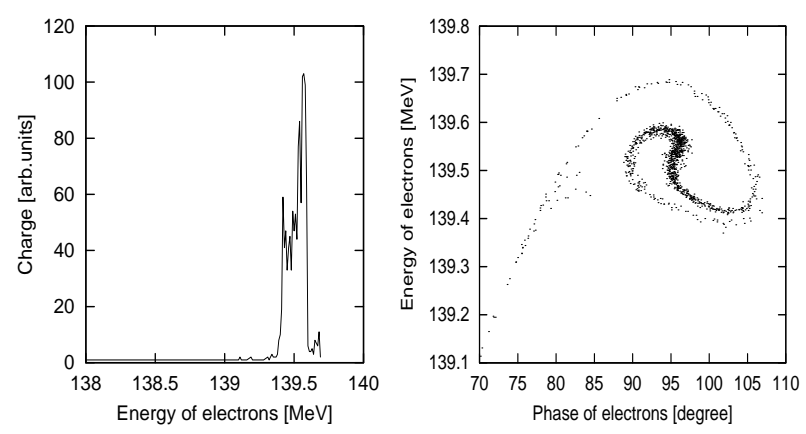

Figure 5 : Final energy spectrum and $(E, \phi)$ distribution of accelerated beam

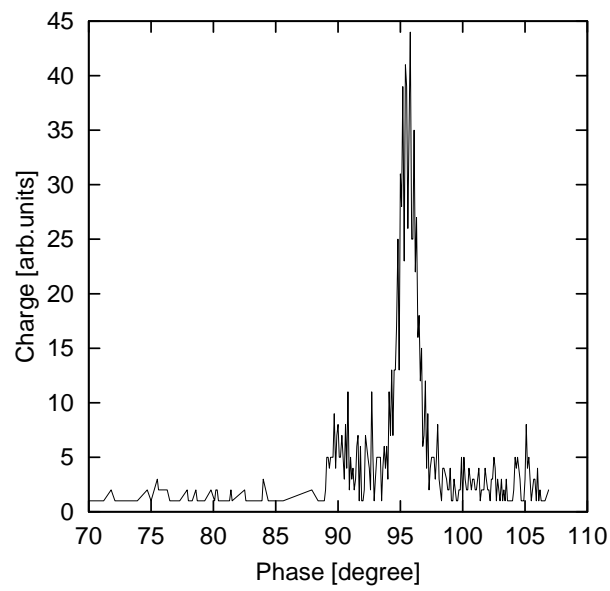

Figure 6 : Final bunch length at $139.55 \mathrm{MeV}$

\section{CONCLUSION}

It has been proved by numerical simulations that the acceleration of ultra-short single-bunch electron beam emitted from a photo cathode RF gun about $5 \mathrm{MeV}$ was achieved. However, in the normal RTM parameter does not permit us conservation of normalized emittance. This may be considered that the reverse field of the first orbit is not perfectly arranged to get better Ex. On the other hand, increase of ey may cause by lack of suitable lattice structures. Hence, we have two ways to upgrade the RTM for the acceleration of ultra-low emittance electron. One is to add focussing magnet for the conservation of
Ey and fine tuning of beam orbit at the reverse field. Other and better way is to be change the system at the first orbit to avoid the reverse magnet and the beam can accelerate through the achromatic orbit.

\section{References}

[1] M. Washio et al., "Femtosecond X-ray Pulse Generation Using a Low Emittance Electron Beam and a High Power Laser", 7th China-Japan Bilateral Symp. On Radiation Chem. Oct. 28, Cengdu, China (1996)

[2] M. Washio et al., "Femtosecond X-rays Produced by Inverse Compton Scattering between a Low Emittance Electron Beam and an Intense Laser Light”, J. Surface Sci. Soc. Jpn., Vol.19 No.2 pp23-29 (1998)

[3] J. Yang et al., "Evaluation of Femtosecond X-rays Produced be Inverse Compton Scattering under Linear and Nonlinear Interactions between a Low Emittance Electron Beam and an Intense Polarised Laser Light", Proc. EPAC'98 Stockholm, pp1082-1084 (1998)

[4] F. Sakai et al., "Development of High Duty Operation RF Photoinjector", Proc. 11th Symposium on Accelerator Sci. and Technology, Harima, Japan pp473-475 (1997)

[5] T. Hori et al., "Simulations of Ultra Short Single Bunch Operation on $150 \mathrm{MeV}$ Microtron", Proc. Epac'98 Stockholm, pp517-519 (1998)

[6] T. Hori et al., "AURORA-2: Compact Advanced SR Ring as an X-ray Source", 4th Int'l Conf. On SR Sources \& 2nd Asian Forum on SR, Kyongju, Korea, pp148-158 (1995) 